\title{
Vascular Reconstructions in Kidney Transplantation
}

\author{
Massimiliano Veroux, Alessia Giaquinta, \\ Giuseppe D'Arrigo, Alberto Davì, \\ Angelo Sanfiorenzo, and Pierfrancesco Veroux
}

\subsection{Introduction}

Kidney transplantation is the preferred treatment for patients in end-stage renal disease, since it improves the overall quality of life and patient survival [1].

The original surgical technique of kidney transplantation was described in 1951 and has only evolved slightly over 60 years [2]. The gold standard surgical procedure is still open and still in either the right or left iliac fossa, with an endto-side anastomosis with the external iliac vessels $[1,2]$. Although the surgical technique has not significantly evolved over the last decades, many improvements have been made in the graft conservation solutions and in the procurement of the aortic cuff and extension of the right renal vein using the inferior vena cava [1, 3-5]. Particularly, the extension of the right renal vein using the inferior vena cava (cava conduit) significantly decreases the rate of renal vein thrombosis and graft loss [3]. Moreover, the use of older donors to increase the donor pool and to face the increasing demand for kidney transplantation has prompted the use of vascular reconstructions to manage the atherosclerotic

\footnotetext{
M. Veroux $(\bowtie) \cdot$ A. Giaquinta · G. D'Arrigo · A. Davì · A. Sanfiorenzo $\cdot$ P. Veroux

Vascular Surgery and Organ Transplant Unit, Department of Surgery, University Hospital of Catania, Catania, Italy

e-mail: veroux@unict.it; pveroux@unict.it
}

aortic and renal arteries. Moreover, kidneys with multiple arteries often require a surgical reconstruction to reduce the number of anastomoses and the cold ischemia time.

Vascular surgery may also be required to manage immediate and late complications of kidney transplantation, mainly the pseudoaneurysm of the renal artery, which is a life-threatening condition, occurring mainly as a consequence of a graft infection.

In this chapter we will describe the main vascular reconstructions adopted in renal transplant surgery.

\subsection{Kidney Transplantation with Multiple Arteries}

The use of grafts with multiple renal arteries (MRA) is a potential risk factor that could impair the outcome of kidney transplantations. Autopsy studies have reported an incidence of $17-35 \%$ MRA grafts, depending on the donor's ethnic origin $[6,7]$. The incidence of multiple arteries in deceased donors is variable: a study among 400 deceased donors reported double arteries in $23 \%$, triple arteries in $4 \%$, and quadruple arteries in $1 \%$ of donors [6-10]. Contradictory results have been reported in literature, and while many studies examining the use of kidneys with multiple renal arteries for renal transplantation did not find that graft survival and function were 
adversely affected by multiple arteries and that vascular reconstructions did not influence recipient creatinine levels or ureteral complication rates [10], many other studies reported an increased incidence of vascular complications, delayed graft function (DGF), and increased warm ischemia time in graft from MRA [3, 6-10].

A recent meta-analysis by Zorgdrager et al. [6] showed that kidney grafts from any type of donor with MRA were associated with a lower 1-year graft survival, a higher complication rate, and an increased frequency of DGF compared to SRA grafts. Both vascular (10.8\%) and urological complications $(5.5 \%)$ are more frequent in MRA grafts. However, no significant differences were observed in the 5-year graft survival and 1- and 5-year patient survival rates. Furthermore, there was a trend toward a longer WIT and higher 1and 5-year creatinine levels in MRA grafts.

The technique of vascular reconstruction may differ significantly when using graft from deceased or living donor. Although ligation of polar arteries supplying $<5-10 \%$ of renal parenchyma may be frequently encountered with an increase in urological complication [11], the presence of MRA often requires vascular reconstruction on bench surgery.

Multiple arteries from kidney grafts from deceased donors may be spatulated together to provide a common outflow or anastomosed endto-side to the main renal artery, even in the case of arterial injury during organ retrieval (Fig. 10.1). Alternatively, in case of $>3$ arteries, a vein patch could be used. These techniques have been also used successfully in dual kidney transplantations (DKT) $[12,13]$. In an interesting study, Veroux et al. [13] reported the results of grafts with multiple arteries in 12 patients who underwent monolateral dual kidney transplantation. In six patients, the accessory arteries were anastomosed end-to-side or side-to-side on the aortic patch. In six patients, with three or more accessory arteries, vascular reconstruction with an inferior vena cava patch was performed, before implanting the kidney (Fig. 10.2). There were no intraoperative complications in the entire series, and there were no immediate vascular complications.
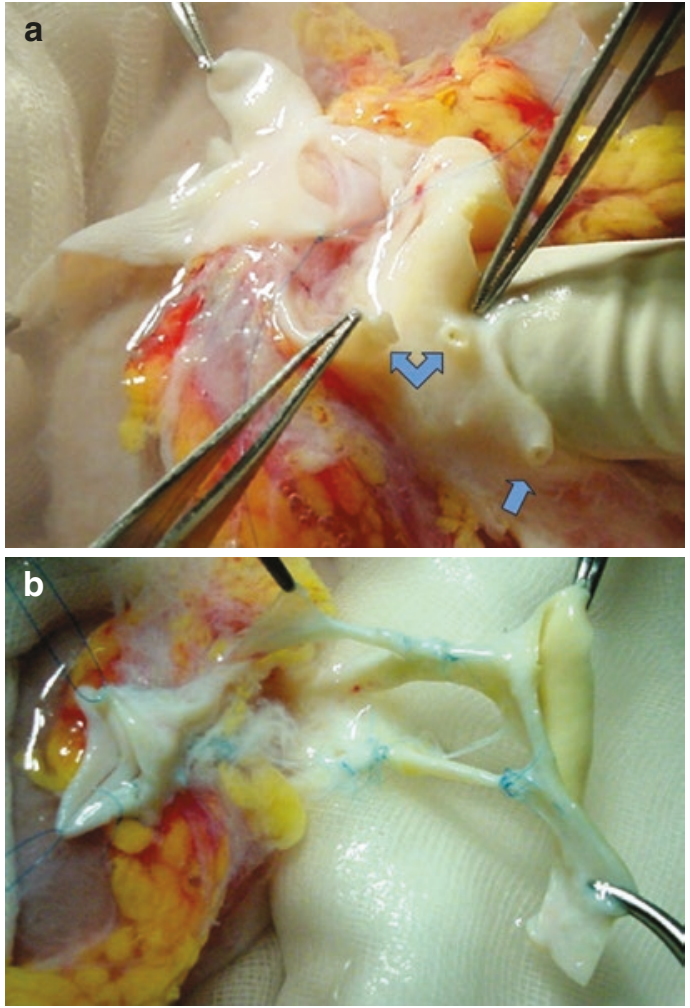

Fig. 10.1 (a) Arterial injury. The renal artery and an accessory renal artery were completely transected from its origin on the aortic patch, during organ retrieval (arrow); (b) the arteries were sutured at bench surgery through an end-to-end anastomosis between the injured renal artery and the aortic patch

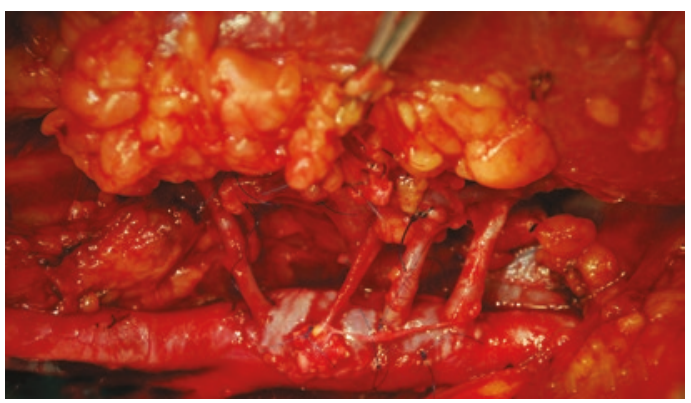

Fig. 10.2 Intraoperative view of reconstructed renal artery on an inferior vena cava patch during a dual kidney transplantation. Four branches are clearly visible

Usually, when feasible, a single anastomosis is preferable to reduce the anastomotic time in the recipient, which has a known wrong effect on graft function. 


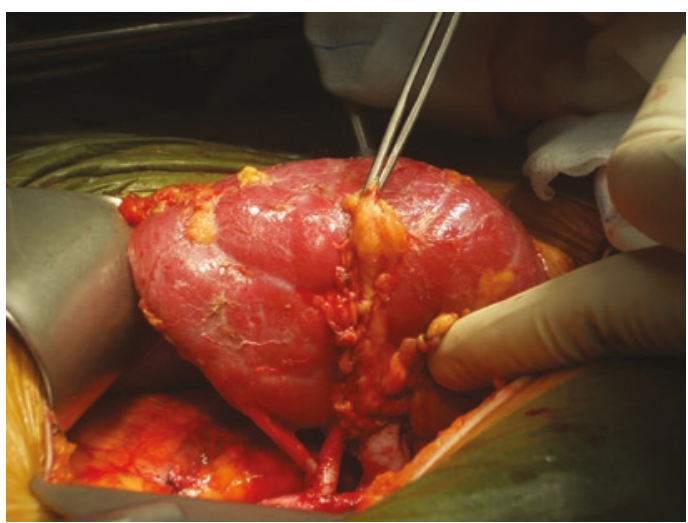

Fig. 10.3 End-to-side anastomosis between an accessory artery and the main renal artery in a living donor kidney transplantation. The kidney was correctly perfused with no signs of parenchymal ischemia

With the increasing demand for organ transplantation due to the increasing number of patients on the waiting list, a growing experience on the use of living donor kidney graft with multiple arteries has accumulated recently, with similar graft and patient outcomes.

Multiple renal artery reconstruction follows the same principles of deceased donor grafts (Fig. 10.3), but recent studies reported excellent outcomes with arterial reconstruction with recipient's own internal iliac artery [14].

\subsection{Vascular Reconstruction in Dual Kidney Transplantation}

Dual kidney transplantation (DKT) of marginal kidneys could offer transplant candidates very satisfactory kidney transplantation in terms of renal function. However, DKT might be considered a major surgical procedure and, in older recipients, has a potentially greater risk of surgical complications compared with single kidney transplantation [12, 13, 15-17].

While different surgical techniques have been described, including the positioning of one kidney for each side extraperitoneally and the positioning of both kidneys intaperitoneally, most transplant centers, to reduce the intraoperative time and the rate of surgical complications, positioned both grafts on the same side $[12,15,16]$.

Ekser et al. [15] analyzed the results of 100 unilateral dual kidney transplantations performed at a single center. When compared with a cohort of single kidney transplantations, surgical complications were similar between the two groups.

A recent review [16] compared the three techniques of bilateral placement, unilateral placement with separate anastomoses, and unilateral placement with patch anastomoses, in dual kidney transplantation, and they reported a similar graft and patient survival among the three groups.

However, in order to reduce the number of anastomoses and the operative time, some transplant centers tried to develop new surgical techniques in dual kidney transplantation. By employing techniques used to conjoin organ vasculature ex vivo, the number of in situ anastomoses is reduced, thereby minimizing operative ischemic time and potential for complications associated with extensive vascular dissection [13, 16, 18-20]. Moreover, these techniques could be used even in those patients with a moderate-to-severe atherosclerosis of the iliac vessels, which is usually a contraindication for dual kidney transplantation [18].

Nghiem [19] proposed a novel technique of transplanting both kidneys en bloc using a single arterial and single venous anastomosis by implanting the renal vein end-to-side alternatively to the left renal vein or on the vena cava. Veroux et al. [18] proposed a new technique of monolateral dual kidney transplantation by joining the two renal veins and the two arteries in one single anastomosis. The technique included a running suture between the posterior margin of the vein of the left kidney and the anterior margin of the right renal vein while the two aortic patches were sutured together (Fig. 10.4).

More recently, Tran et al. [20] described an alternative technique of dual kidney transplantation in 22 patients by using the donor's common iliac artery with its internal and external branches for performing an interposition Y-graft to join the left and the right renal artery of the graft. The suprarenal vena cava was oversewn in a running 

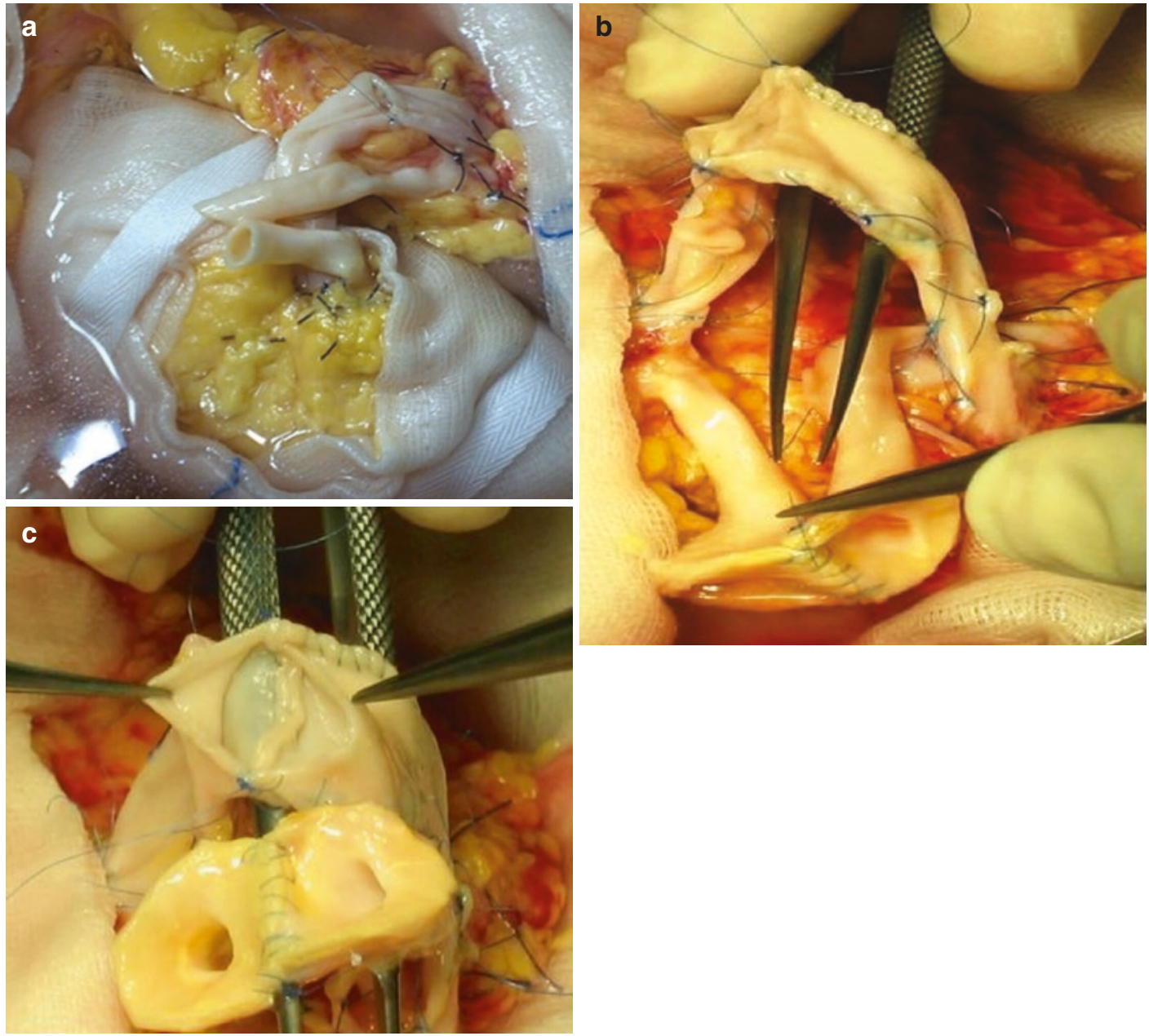

Fig. 10.4 Technique of cojoining renal vessels in dual kidney transplantation. After elongation of the right renal vein with the vena cava, (a) the two renal veins were sutured together, joining the posterior margin of the vein

fashion, and the distal part of the inferior vena cava was used for anastomosis.

\subsection{Renal Aneurysm and Pseudoaneurysm}

Renal artery aneurysms (RAAs) are defined as dilation of all three layers of the arterial wall $>1 \mathrm{~cm}$ in diameter [21-23]. Renal artery aneurysms (RAAs) are very uncommon, with a widely accepted incidence of $0.1 \%$ in the general population $[21,22]$. Approximately $75 \%$ of true RAAs are saccular in shape, and $25 \%$ are found to be fusiform. RAAs most commonly affect the

of the left kidney with the anterior margin of the right renal vein. Then (b) the two aortic patches were sutured together. (c) Final result with the joined arteries and veins

main bifurcation but can be located anywhere in the arterial tree [21].

In a kidney transplant setting, the evidence of an aneurysm of the main renal artery or one of its branches is an uncommon finding but usually requires surgical repair to allow for its use for kidney transplantation. The most frequently adopted technique is similar to the ex vivo technique adopted in patients with an aneurysm of native kidneys, and the technique involves a resection of the renal artery aneurysm and a suture of the arterial wall [23].

Renal pseudoaneurysm (RPA) forms as a consequence of arterial wall injury: the kidney tissue surrounding the site of injury exerts a compres- 
sive force to slow or completely stop the bleeding, so that a hematoma forms around the arteriotomy [24]. The subsequent clot retraction and the reactive fibrosis form a structure resembling an aneurysmal sac, which can be eventually connected with the intraluminal space, leading to expansion of the pseudoaneurysmal sac and pain or rupture.

Renal pseudoaneurysm is a life-threatening condition because it involves an arterial injury that is occluded solely by hematoma and connective tissue. The degree of bleeding depends on the delicate balance between the tamponade effect of the surrounding hematoma and connective tissue and the intraluminal hydrostatic pressure changes [24]. Anastomotic pseudoaneurysm of renal allograft is a rare and life-threatening vascular complication after kidney transplantation, occurring in $<1 \%$ of cases. Intrarenal pseudoaneurysms are more common and usually resolve spontaneously. In contrast, extrarenal pseudoaneurysms are related to a mycotic infection at the site of anastomosis and usually occur in the early weeks after transplantation as a result of anastomotic defects, such as suture rupture, anastomotic leakage, vessel wall ischemia, or arterial dehiscence caused by local infection. In the case of infection, the risk of pseudoaneurysm rupture and graft loss is high. Fungal infection may originate from the donor or, more likely, from exogenous sources leading to contamination during graft handling and implantation [23].

Pseudoaneurysms of the transplant renal artery are usually asymptomatic, and the diagnosis is mainly incidental, during follow-up outpatient visits. Although rare, these complications may have a high risk of anastomotic leakage or arterial wall rupture, resulting in hemorrhagic shock and graft loss [23].

Surgical repair of transplant renal artery pseudoaneurysm is challenging and often requires specialized treatment, due to the inflammatory tissue surrounding the renal artery, and, in most cases, transplant nephrectomy may ultimately be required: in the series of six patients with anastomotic pseudoaneurysm of the renal transplant artery reported by Bracale et al. [25], a transplant nephrectomy was needed in all but one patient.
However, in a recent report, Ardita et al. [26] reported a successful pseudoaneurysm repair with in situ bypass (Fig. 10.5) between the internal iliac artery and the renal artery, while the common iliac artery was revascularized through an autologous vein bypass between the proximal external iliac artery and the common femoral artery.
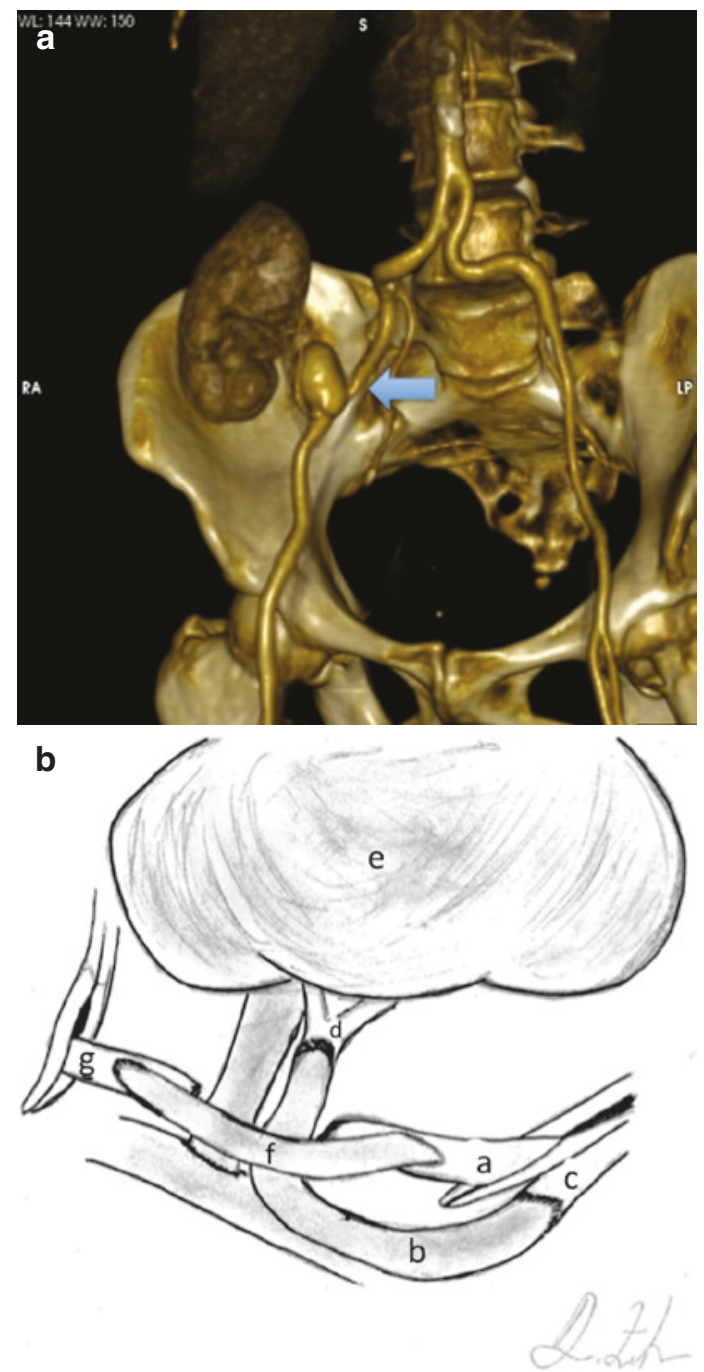

Fig. 10.5 Anastomotic pseudoaneurysm of the renal transplant artery. (a) Computed tomography angiography demonstrated a 3-cm anastomotic pseudoaneurysm of the graft. (b) Scheme of surgical procedure after resection of the anastomotic pseudoaneurysm. The external iliac artery (a) was dissected, and a bypass $(c)$ was performed between the internal iliac artery $(b)$ and the renal artery $(d)$ of kidney transplantation $(e)$. External iliac artery was revascularized through a bypass between proximal external iliac artery and common femoral artery $(f)$ 


\subsection{Summary and Conclusions}

Vascular reconstructions are often required in the kidney transplant setting.

Multiple arteries are frequently encountered and usually require a surgical reconstruction to minimize the number of vascular anastomoses. Renal artery aneurysm and pseudoaneurysms are rare but potentially life-threatening and require a challenging surgical repair. Vascular reconstruction techniques may also be used in dual kidney transplantation to reduce the operative ischemic time and potential for complications associated with extensive vascular dissection.

\section{References}

1. Veroux M, Corona D, Veroux P. Kidney transplantation: future challenges. Minerva Chir. 2009;64(1):75-100.

2. Kuss R, Teinturier J, Milliez P. Some attempts at kidney transplantation in man. Mem Acad Chir (Paris). 1951;77:755.

3. Bessede T, Droupy S, Hammoudi Y, et al. Surgical prevention and management of vascular complications of kidney transplantation. Transpl Int. 2012;25:994-1001.

4. Benoit G, Jaber N, Moukarzel M, et al. Incidence of vascular complications in kidney transplantation: is there any interference with the nature of the perfusion solution? Clin Transpl. 1994;8:485.

5. Fung LC, McLorie GA, Khoury AE, Churchill BM. Donor aortic cuff reduces the rate of anastomotic arterial stenosis in pediatric renal transplantation. J Urol. 1995; 154:909.

6. Zorgdrager M, Krikke C, Hofker SH, et al. Multiple renal arteries in kidney transplantation: a systematic review and meta-analysis. Ann Transplant. 2016;21:469-78.

7. Khamanarong K, Prachaney P, Utraravichien A, et al. Anatomy of renal arterial supply. Clin Anat. 2004;17:334-6.

8. Bessede T, Droupsy S, Hammoudi Y, et al. Surgical prevention and management of vascular complications of kidney transplantation. Transpl Int. 2012;25:994-1001.

9. Osman Y, Skokeir A, Ali-el-Dein B, et al. Vascular complications after live donor renal transplantation: study of risk factors and effect on graft and patient survival. J Urol. 2003;169:859-62.

10. Hellegering J, Visser J, Kloke HJ, et al. Deleterious influence of prolonged warm ischemia in liv- ing donor kidney transplantation. Transplant Proc. 2012;44:1222-6.

11. Kok NF, Dols LF, Hunink MG, et al. Complex vascular anatomy in live kidney donation: imaging and consequences for clinical outcome. Transplantation. 2008;85:1760-5.

12. Veroux M, Corona D, Gagliano M, et al. Monolateral dual kidney transplantation from marginal donors. Transplant Proc. 2007;39(6):1800-2.

13. Veroux M, Giuffrida G, Ekser B, et al. Vascular reconstruction in monolateral dual kidney transplantation with multiple arteries. Clin Transpl. 2012;26(3):443-6.

14. Hiramitsu T, Futamura K, Okada M, et al. Impact of arterial reconstruction with recipient's own internal iliac artery for multiple graft arteries on living donor kidney transplantation: strobe study. Medicine (Baltimore). 2015;94(43):e1811.

15. Ekser B, Furian L, Broggiato A, et al. Technical aspects of unilateral dual kidney transplantation from expanded criteria donors: experience of 100 patients. Am J Transplant. 2010;10(9):2000-7.

16. Cocco A, Shahrestani S, Cocco N, et al. Dual kidney transplant techniques: a systematic review. Clin Transpl. 2017;31(8):28544075.

17. Renaud S, Tinsit MO, Rabant M, et al. Dual kidney transplantation: is it worth it? Transplantation. 2017;101:488-97.

18. Veroux P, Giuffrida G, Cappellani A, et al. Two-asone monolateral dual kidney transplantation. Urology. 2011;77:227-30.

19. Nghiem DD. Simultaneuos double adult kidney transplantation using single arterial and venous anastomoses. Urology. 2006;67:1076-8.

20. Tran KC, Li D, Taqi A, et al. Dual en bloc technique for adult renal transplantation. Clin Transpl. 2017;31(8):28523715.

21. Orion KC, Abularrage CJ. Renal artery aneurysms: movement toward endovascular repair. Semin Vasc Surg. 2013;26:226-32.

22. Colemna DM, Stanley JC. Renal artery aneurysms. J Vasc Surg. 2015;62:779-85.

23. Veroux P, Giaquinta A, Zerbo D, De Marco E, Sanzone A, Veroux M. Surgery for the renal arteries. In: Chiesa R, Setacci C, Castelli PM, editors. Tip and tricks in open vascular surgery. Torino: Edizioni Minerva Medica; 2017. p. 93-100. ISBN: 978-88-7711-885-1.

24. Ngo TC, Lee J, Gonzalgo ML. Renal pseudoaneurysm: an overview. Nat Rev Urol. 2010;7:619-25.

25. Bracale UM, Santangelo M, Carbone F, et al. Anastomotic pseudoaneurysm complicating renal transplantation: treatment options. Eur J Vasc Endovasc Surg. 2010;39:565-8.

26. Ardita V, Veroux M, Zerbo D, et al. Non-mycotic anastomotic pseudoaneurysm of renal allograft artery. Case report. Ann Ital Chir. 2016;87:27319817. 